\title{
Pyruvate Accumulation and Development of Thiamine Deficiency in Cultures of Proteus vulgaris
}

\author{
By F. L. JACKSON* \\ National Institute for Medical Research, Mill Hill, London, N.W. 7 and \\ King's College Hospital Medical School, Denmark Hill, London, S.E. 5
}

SUMMARY: The accumulation of pyruvate in cultures of Proteus vulgaris grown with limiting concentrations of nicotinic acid has been investigated. Nicotinic acid-deficient organisms remaining in contact with a lactate medium under conditions where pyruvate accumulation is occurring gradually become deficient in thiamine. The thiamine content of organisms at various stages of growth was estimated, and a relationship between thiamine content and length of lag phase found. Maximum rates of pyruvate oxidation were obtained in organisms which were not deficient in either nicotinic acid or thiamine. The possible metabolic significance of these findings is discussed.

As shown by Jackson \& Copping (1952) the growth curve of Proteus vulgaris in a defined medium, with lactate as sole carbon source and limiting concentrations of nicotinic acid, has a characteristic form, the phase of exponential synthesis being followed by a phase of arithmetic linear growth. A preliminary account of pyruvate accumulation in the medium and of the development of thiamine deficiency in the organisms was given by Jackson $(1952 a, b)$; the present paper presents a detailed account of some of these observations and of additional findings.

\section{METHODS}

The organism, medium, conditions of incubation, opacity measurements, dryweight determinations and measurements of oxygen uptake were as described by Jackson \& Copping (1952). Thiamine, in freshly prepared aqueous solution, was added to the medium as required.

Thiamine assays. Organisms from suitable culture samples $(40$ or $80 \mathrm{ml}$. according to the concentration of the culture) were collected by centrifugation at $12,500 \mathrm{rev} . / \mathrm{min}$. and washed twice with glass-distilled water. They were resuspended in $5 \mathrm{ml} .0 .05 \mathrm{M}$-acetate buffer $(\mathrm{pH} 4.5)$ and were killed by heating in a boiling-water bath for $\mathbf{2 0} \mathrm{min}$. Digestion with a papain and taka-diastase mixture and microbiological assay with Lactobacillus fermentum were as described by Barton-Wright (1946). This method was used for estimation of the thiamine content of the supernatant fluids of centrifuged samples.

The pyruvate content of the medium was estimated by the method of $\mathbf{L u}$ (1939). In early experiments the results obtained by Lu's method were checked manometrically, using the technique described by Umbreit, Burris \&

* At present, Cowburn Research Fellow, King's College Hospital Medical School, Denmark Hill, London, S.E. 5. 
Stauffer (1949), and no significant difference between the two methods was found. Values are expressed as pyruvic acid.

In experiments on the lag phase of cultures, the following substances were used: $\beta$-alanine, $p$-aminobenzoic acid, folic acid, pyridoxin, uracil, coenzyme $\mathrm{I}$, xanthine, riboflavin, calcium-D-pantothenate, 4-methyl-5-hydroxyethylthiazole and 2-methyl 4-amino-5-ethoxymethylpyrimidine. Fresh solutions were prepared and used as required.

Chromatography. In experiments on the accumulation of pyruvate, both in the lag phase and during the late stages of growth, the keto-acid was identified chromatographically; the method of Altmann, Crook \& Datta (1951) was used. Pyruvate was the only substance detected.

Coenzyme I. A preparation of coenzyme was made from baker's yeast by the method of Page (in Umbreit et al. 1949).

Cocarboxylase. Cocarboxylase was obtained from Roche Products Ltd., Welwyn Garden City, Herts, who also kindly provided the samples of 4-methyl-5-hydroxyethylthiazole and 2-methyl 4-amino-5-ethoxymethylpyrimidine.

Nicotinic acid concentrations. In the medium used, concentrations of 0.02 , 0.03 or $0.04 \mu \mathrm{g}$. nicotinic acid $/ \mathrm{ml}$. were growth limiting, and on the linear portion of a dose-response curve. Concentrations of nicotinic acid of about $0.2 \mu \mathrm{g} . / \mathrm{ml}$. were just in excess of maximum requirements at the lactate concentration used. In experiments where the nicotinic acid concentration is described as 'an excess', the concentration of nicotinic acid was $2 \cdot 4 \mu \mathrm{g} . / \mathrm{ml}$.

\section{RESULTS}

Pyruvate accumulation in the medium

During the phase of exponential growth which occurred before the nicotinic acid concentration became limiting, pyruvate concentrations of $10-15 \mu \mathrm{g} . / \mathrm{ml}$. were regularly found in the medium, but at the onset of linear growth the pyruvate concentration began to rise, and this accumulation continued during the later growth period and after growth had ceased (Fig. 1). During the linear growth phase, the rate of pyruvate accumulation increased with time, but when growth had ceased, the rate of pyruvate accumulation remained constant. The effect of adding nicotinic acid to a culture in the linear growth phase is shown in Fig. 2. There was a prompt decrease in pyruvate concentration and restoration of exponential growth. When thiamine or cocarboxylase to final concentration $1.6 \times 10^{-4} \mathrm{M}$ and not nicotinic acid, was added during the phase of linear growth, further accumulation of pyruvate was not prevented, and there was no measurable change in growth rate. The effect of coenzyme I was similar to that of nicotinic acid.

The cultures used were fully grown after about $30 \mathrm{hr}$. of incubation, and by the time they were $48 \mathrm{hr}$. old they had accumulated more than $3 \mathrm{mg}$. pyruvate $/ \mathrm{ml}$. (expressed as pyruvic acid). A $48 \mathrm{hr}$. culture of this kind was divided into five parts and the effects of adding nicotinic acid, thiamine, coenzyme $\mathrm{I}$ and cocarboxylase in final concentrations of $2 \times 10^{-5} \mathrm{M}$ were 
observed. Nicotinic acid and coenzyme I both promoted growth associated with a decrease in pyruvate concentration; thiamine and cocarboxylase brought about a definite but much less rapid decrease, and cocarboxylase was about twice as effective as thiamine on a molar basis.

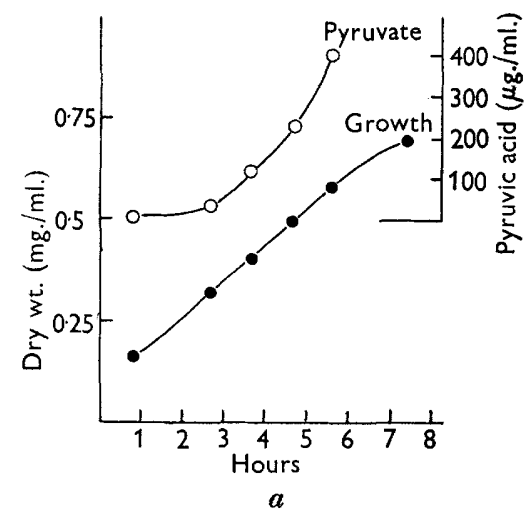

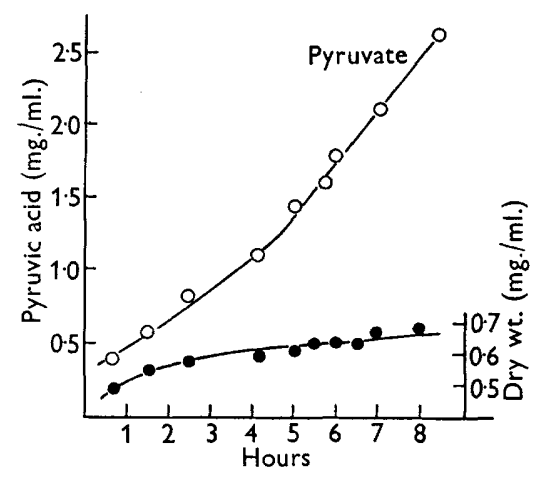

$b$

Fig. 1. (a) The early stages of pyruvate accumulation during the linear growth phase.

(b) Continuation of pyruvate accumulation after growth had practically ceased.

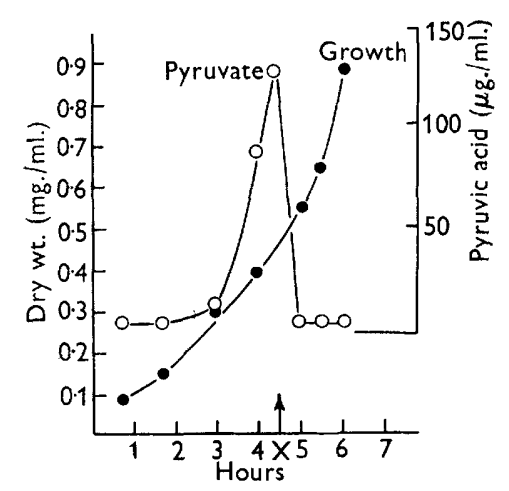

Fig. 2. Effects of adding more nicotinic acid to a culture in the linear growth phase. Nicotinic acid to a final concentration of $2 \cdot 4 \mu \mathrm{g} . / \mathrm{ml}$. was added at X.

As would be expected, when growth was limited by lactate concentration and not by nicotinic acid concentration, no pyruvate accumulation was found. When nicotinic acid was limiting, the pyruvate concentrations found at $72 \mathrm{hr}$. increased as the amount of lactate in excess of growth requirements was increased. In a series of cultures with lactate concentrations of from 1 to $8 \mathrm{mg} . / \mathrm{ml}$., and all containing $0.04 \mu \mathrm{g}$. nicotinic acid $/ \mathrm{ml}$., no pyruvate accumulation was found in cultures with 1, 2, 3 or $4 \mathrm{mg}$. lactate $/ \mathrm{ml}$., and in these lactate was limiting. When lactate concentrations in excess of $4 \mathrm{mg} . / \mathrm{ml}$. were used, pyruvate accumulation occurred, and the concentrations found were roughly proportional to the excess of lactate. 


\section{Lag phase phenomena}

For investigation of the lag phase of the organisms on transference to fresh medium, organisms were harvested at different times during and after growth and were washed twice with glass-distilled water. They were then re-suspended
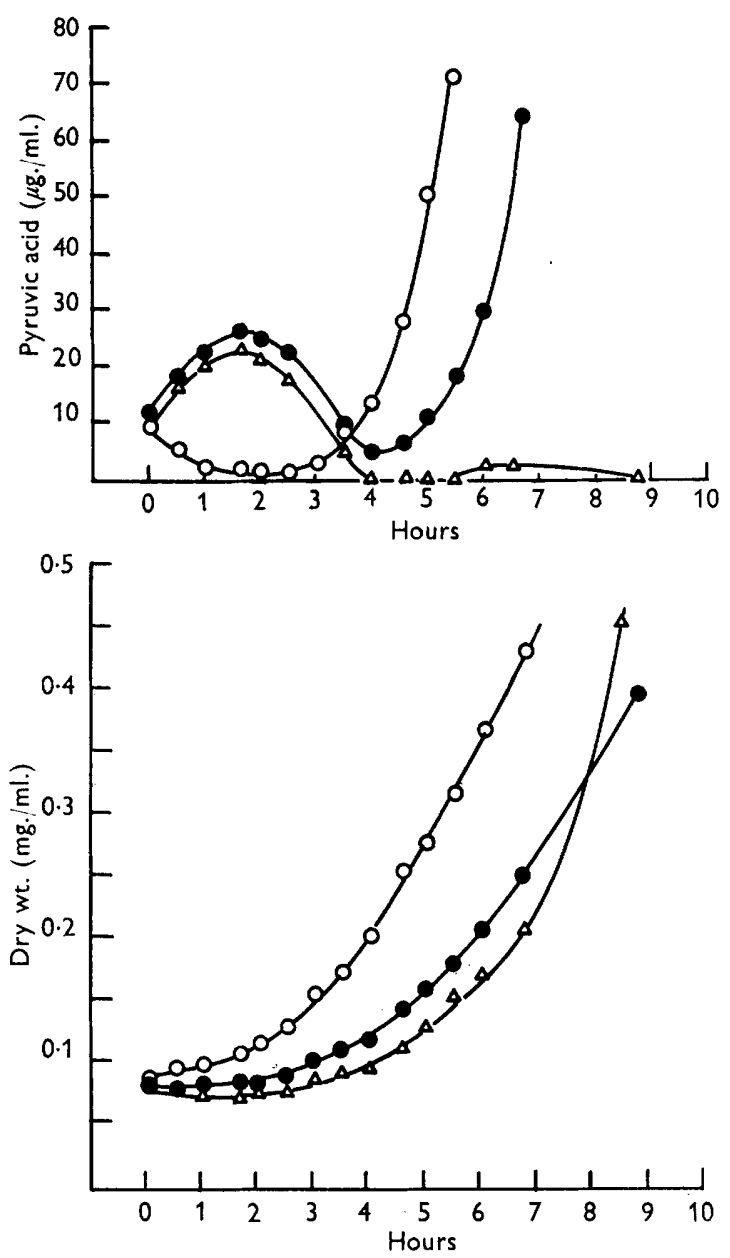

Fig. 3. Curves of growth and pyruvate concentrations in cultures inoculated at time 0 with thiamine-deficient organisms. Three cultures were used, and are represented respectively by circles, dots and triangles in both pyruvate and growth curves. $0.03 \mu \mathrm{g}$. nicotinic acid $/ \mathrm{ml}$; $\bigcirc-0,0.03 \mu \mathrm{g}$. nicotinic acid $/ \mathrm{ml} .+10^{-5} \mathrm{M}$-thiamine; $\triangle-\triangle, \mathbf{2 \cdot 4} \mu \mathrm{g}$. nicotinic acid $/ \mathrm{ml}$.

in fresh medium to a concentration equivalent to $c .0 .03 \mathrm{mg}$. dry wt. organisms $/ \mathrm{ml}$., and samples were taken at intervals for opacity and pyruvate estimations.

The addition of thiamine + nicotinic acid to the medium did not affect the lag phase of organisms harvested during the linear phase of growth or at 
about the time of cessation of growth. With organisms collected at these times the lag phase was short (less than $30 \mathrm{~min}$.).

With organisms collected 8-12 hr. after the cessation of growth, however, the findings were different; they are shown in Fig. 3. The addition of thiamine to final concentration $10^{-5} \mathrm{M}$ strikingly shortened the lag phase, and sometimes practically abolished it. Pyruvate estimations during the lag phase showed that, in cultures without added thiamine, an initial increase of pyruvate concentration of about $25 \mu \mathrm{g} . / \mathrm{ml}$. was followed by a decrease. Growth in the cultures began when the pyruvate concentration had decreased to $c .10 \mu \mathrm{g} . / \mathrm{ml}$. The addition of an excess of nicotinic acid during the lag phase did not prevent this pyruvate accumulation, and of numerous substances tested only thiamine and cocarboxylase were effective in preventing pyruvate accumulation and in shortening lag. The ineffective factors tested were $\beta$-alanine, $p$-aminobenzoic acid, folic acid, pyridoxin, uracil, xanthine, riboflavine and calcium-Dpantothenate. Pyruvate accumulating during the lag phase was identified chromatographically.

Concentrations of added thiamine ranging from $2 \times 10^{-5} \mathrm{M}$ to $2 \times 10^{-8} \mathrm{M}$ had approximately similar effects on the lag phase, with a starting inoculum equivalent to $150 \mu \mathrm{g}$. dry wt. organisms $/ \mathrm{ml}$. Two possible precursors of thiamine, 4-methyl-5-hydroxyethylthiazole and 2-methyl-4-amino-5-ethoxymethylpyrimidine were tested separately and in combination for effects on the lag phase, but were found to be ineffective.

When cultures to which thiamine had been added during the lag phase were followed during their total period of growth, pyruvate was found to accumulate during the linear phase, when the nicotinic acid supply was growthlimiting, as in cultures without added thiamine. The mean generation time in cultures to which thiamine was added initially was rather less, during the exponential phase, than in cultures which had been supplied with nicotinic acid alone, and growth during the linear phase was also slightly faster (Fig. 3). These effects on growth rate were only seen when thiamine was added at the time of inoculating the cultures, and not when it was added after nicotinic acid had become limiting.

\section{Effects of thiamine on $\mathrm{O}_{2}$ uptake}

The alterations in growth and pyruvate accumulation described were accompanied by corresponding effects on oxygen uptake of suspensions of washed organisms. The addition of thiamine to washed suspensions of organisms collected during the linear growth phase or shortly after cessation of growth did not increase the rate of $\mathrm{O}_{2}$ uptake with pyruvate as substrate and excess of added nicotinic acid. The rate of oxygen uptake of organisms collected 8-12 hr. after the cessation of growth was, however, strikingly greater when thiamine + nicotinic acid were added than when only nicotinic acid was added. Cocarboxylase was slightly more active than thiamine, on a molar basis (Fig. 4). 


\section{The thiamine content of organisms}

The thiamine content of organisms collected at different times during growth in media containing either limiting concentrations or an excess of nicotinic acid was estimated. Organisms growing exponentially in medium with optimal nicotinic acid concentrations were found to contain $40-50 \mu \mathrm{g}$. thiamine/g. dry wt. organisms. Under these conditions, i.e. with excess of nicotinic acid and limiting lactate concentration, this concentration of thiamine

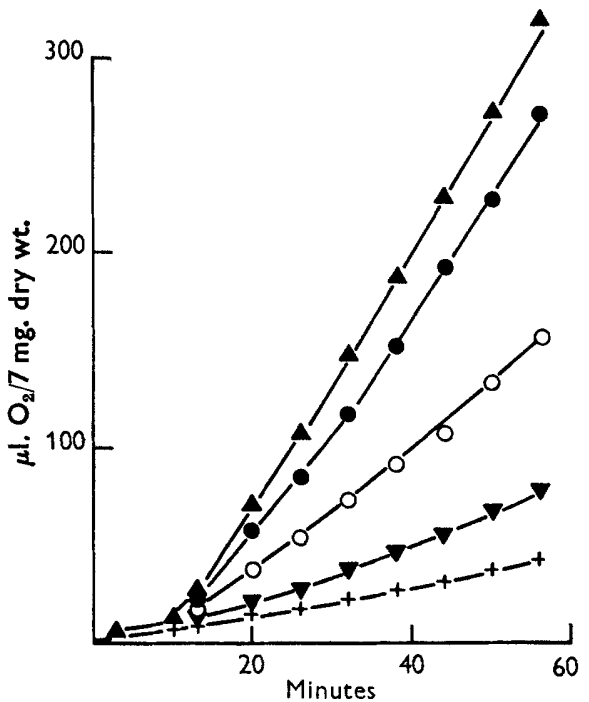

Fig. 4. Oxygen uptake, with pyruvate as substrate, of thiamine-deficient organisms with added nicotinic acid, thiamine and cocarboxylase. +-+ , no additions; $\nabla-\nabla$, cocarboxylase; $\bigcirc-O$, nicotinic acid; - , nicotinic acid + thiamine; $\boldsymbol{\Delta}-\boldsymbol{\Delta}$, nicotinic acid + cocarboxylase. Final volume in flasks was $3.0 \mathrm{ml}$. Pyruvate 0.036 ; concentration of added factors, 8.3 $\times 10^{-6} \mathrm{M} ; \mathrm{MgSO}_{4}, 2 \times 10^{-4} \mathrm{M}$ present in each flask; phosphate $0.03 \mathrm{M} \cdot \mathrm{pH7} \cdot 4$. The thiamine or cocarboxylase was placed in the main compartment of the flasks. Nicotinic acid was added from the side-arm $5 \mathrm{~min}$. after the zero reading. The centre well contained a folded $2 \mathrm{~cm} .^{2}$ of filter paper and $0.1 \mathrm{ml} .20 \% \mathrm{KOH}$.

in the organisms was maintained for at least $48 \mathrm{hr}$. after cessation of growth. Organisms collected from cultures with limiting concentrations of nicotinic acid contained about 30-40 $\mu \mathrm{g}$. thiamine/g. dry wt. organisms at the end of the linear growth phase, and during $12 \mathrm{hr}$. after the cessation of growth the content fell to 10-12 $\mu \mathrm{g}$. thiamine/g. dry wt. organisms. When cultures were grown with limiting nicotinic acid concentrations but with $0 \cdot 2 \mu \mathrm{g}$. thiamine $/ \mathrm{ml}$. present initially, the thiamine content of the organisms remained high during and after the linear phase of growth. In a culture of this kind, the concentration of thiamine in the organisms after $48 \mathrm{hr}$. was $60 \mu \mathrm{g}$. $/ \mathrm{g}$. dry wt. organisms. Thiamine estimations on organisms and supernatant fluids from the culture showed that the total organism + supernatant fluid thiamine amounted to only $c .35 \%$ of that initially added. The thiamine concentration in the supernatant fluid of the centrifuged culture at the end of the experiment was $0.035 \mu \mathrm{g} . / \mathrm{ml}$. 
Thiamine synthesis during lag phase

Organisms which contained initially $15 \mu \mathrm{g}$. thiamine/g. dry wt. were suspended in fresh medium, with an excess of nicotinic acid, to a concentration equivalent to $\mathbf{0 \cdot 2 6} \mathrm{mg}$. dry wt. organism $/ \mathrm{ml}$. Pyruvate accumulation and opacity changes were followed during the lag phase, and a sample of organisms for thiamine estimation was collected when the pyruvate concentration was falling and growth was just beginning. During the lag phase the thiamine content of the organisms rose from the initial $15 \mu \mathrm{g}$. $/ \mathrm{g}$. dry wt. organism to $40 \mu \mathrm{g} . / \mathrm{g} . \mathrm{dry}$ wt.

\section{Thiamine uptake by cells}

Organisms were collected from cultures which had been grown for $48 \mathrm{hr}$. with a limiting concentration of nicotinic acid $(0.02 \mu \mathrm{g} . / \mathrm{ml}$.) and with an excess of nicotinic acid. They were re-suspended in $10 \mathrm{ml}$. vol. of $0 \cdot 2 \mathrm{M}-$ phosphate buffer ( $\mathrm{pH} \mathrm{7.3)}$ to a concentration equivalent to $1.8 \mathrm{mg}$. dry wt. organisms $/ \mathrm{ml}$. and $2 \mathrm{ml}$. of a thiamine solution containing $1 \mu \mathrm{g}$. thiamine $/ \mathrm{ml}$. was added to each. The suspensions were shaken at $37^{\circ}$ for $10 \mathrm{~min}$. and were then centrifuged. The organisms were washed three times with glass-distilled water and their thiamine content determined. Simultaneous thiamine estimations were made on samples from the same cultures which were not exposed to thiamine after collection. The thiamine content of the organisms grown with an excess of nicotinic acid was $50 \mu \mathrm{g}$. $/ \mathrm{g}$. dry wt. organism and this was not increased by exposure to thiamine in the concentration used. The thiamine content of the organisms grown with limiting nicotinic acid concentration was $12 \mu \mathrm{g}$. thiamine/g. dry wt. organism and after exposure to thiamine this rose to $55 \mu \mathrm{g} . / \mathrm{g}$. dry wt. organism.

\section{Effects of incubation with lactate on thiamine content of organisms}

Three $30 \mathrm{ml}$. samples (A, B, C) of a culture which had grown for $24 \mathrm{hr}$. with excess of nicotinic acid were collected and centrifuged. The thiamine content of sample $\mathbf{A}$ was estimated at this stage. The sample $\mathbf{B}$ was resuspended in $40 \mathrm{ml}$. 0.05 M-phosphate buffer $(\mathrm{pH} 7 \cdot 4)+10 \mathrm{ml}$. water, and was incubated overnight on the shaker. Sample C was resuspended in $40 \mathrm{ml} .0 .05 \mathrm{M}$-phosphate buffer $(\mathrm{pH} 7 \cdot 4)+10 \mathrm{ml} .0 \cdot 5 \mathrm{~m}$-lactate and incubated overnight on the shaker. The organisms from suspensions $\mathrm{B}$ and $\mathrm{C}$ were collected and their thiamine content determined for comparison with $\mathrm{A}$. The thiamine concentrations found were ( $\mu \mathrm{g}$. thiamine/g. dry wt. organism), respectively: A, 33; B, 30; C, 19. Pyruvate had accumulated in C.

\section{Crystallization of pyruvic 2:4-dinitrophenylhydrazone from cultures}

Pyruvic 2:4-dinitrophenylhydrazone could easily be crystallized from culture medium which had accumulated $3 \mathrm{mg}$. pyruvate $/ \mathrm{ml}$. To $400 \mathrm{ml}$. culture fluid after centrifugation was added about $90 \%$ of the calculated required amount (on the basis of pyruvate content of the medium) of concentrated 2:4-dinitrophenylhydrazone in $2 \mathrm{~N}-\mathrm{HCl}$; the pyruvic 2:4-dinitrophenylhydrazone was precipitated. The precipitate was collected on a sintered 
glass filter and was washed twice with $2 \mathrm{~N}-\mathrm{HCl}$. The crude pyruvic 2:4-dinitrophenylhydrazone was dissolved in a minimal amount of ethyl acetate and transferred to a separating funnel. The ethyl acetate solution was extracted with a minimal amount of $10 \%(\mathrm{w} / \mathrm{v})$ sodium carbonate solution, and the sodium carbonate layer collected. The pyruvic 2:4-dinitrophenylhydrazone was precipitated from this by slow addition of $4 \mathrm{~N}-\mathrm{HCl}$, and the precipitate was collected by filtration using a sintered glass filter. The precipitate was washed 5 times with $\mathrm{N}-\mathrm{HCl}$, redissolved in a minimal quantity of warm ethyl acetate, heated cautiously over a water-bath, and when it had dissolved, the solution was filtered through a Whatman no. 54 filter paper. Crystallization occurred from the filtrate. The solid material was recrystallized several times from ethyl acetate and was dried in vacuo over $\mathrm{H}_{2} \mathrm{SO}_{4}$. The melting-point was found to be $217^{\circ}$; a mixed melting-point with a known pure sample was the same. The nitrogen content corresponded to the expected value of $20.9 \%$.

\section{Results with other strains of Proteus vulgaris}

Five other nicotinic acid-requiring strains of Proteus vulgaris were investigated; in all of them similar phenomena occurred during the lag phase and linear and terminal growth phases.

\section{DISCUSSION}

The accumulation of pyruvate during the phase of linear growth in cultures of Proteus vulgaris with growth-limiting nicotinic acid concentrations is quickly reversed by the addition of more nicotinic acid or coenzyme I (diphosphopyridine nucleotide). The organisms at this time are not strikingly deficient in thiamine, although their content is $c .10-15 \mu \mathrm{g} . / \mathrm{g}$. dry wt. organism below that found in cultures grown with excess nicotinic acid. The presence of $10^{-5} \mathrm{M}$-thiamine in the medium initially does not prevent pyruvate accumulation during the linear phase, and under these conditions the thiamine content of the organisms exceeds $50 \mu \mathrm{g}$./g. dry wt. organism. Throughout the linear growth phase the concentration of nicotinic acid in the developing mass of organisms must be diminishing, since all the available nicotinic acid is in the organisms and at the same time growth is continuing (Jackson \& Copping, 1952). The thiamine concentration in the organisms remains in excess of $30 \mu \mathrm{g} . / \mathrm{g}$. dry wt. organism even in the absence of added thiamine.

In the stationary phase, the organisms which are too deficient in nicotinic acid for growth to occur are in contact with a medium in which the concentration of pyruvate is steadily increasing. Under these conditions, the organisms become thiaminc-deficient and the addition of nicotinic acid alone to a pyruvate-containing suspension of washed organisms which are deficient in both nicotinic acid and thiamine is followed by a marked increase in rate of oxygen uptake. However, when thiamine or cocarboxylase is added with nicotinic acid the rate of oxygen uptake is increased still further and may be double that observed with nicotinic acid alone. The addition of thiamine or cocarboxylase alone causes only a slight increase in rate of oxygen uptake. 
These effects of added thiamine or cocarboxylase are not seen with organisms collected at the end of the linear phase of growth or early in the stationary phase, when the cell concentration of thiamine in the organisms is about $30 \mu \mathrm{g} . / \mathrm{g}$. dry wt. organism. The effects of thiamine or cocarboxylase on the lag phase of organisms which have been washed and transferred to fresh medium parallel the effects on oxygen uptake. The lag phase experiments emphasize the fact that, in these cultures, the onset of growth is related to the increased ability of the organisms to utilize pyruvate, which is dependent on adequate concentrations of thiamine and nicotinic acid in the organisms.

The development of thiamine deficiency during the stationary phase within a similar period does not occur when lactate, and not nicotinic acid concentration, is limiting growth. Under these conditions the lactate is completely utilized, and the organisms do not become deficient in nicotinic acid. Accumulation of pyruvate does not occur since there is not an excess of lactate during the stationary phase, and the organisms do not remain in contact during this phase with a pyruvate solution.

During the lag phase of thiamine-deficient organisms in the absence of added thiamine but with added nicotinic acid, thiamine is synthesized by the organisms and growth does not occur until concentrations of thiamine of c. $40 \mu \mathrm{g} . / \mathrm{g}$. dry wt. organisms have been reached. As thiamine is synthesized, the ability to metabolize pyruvate is increased, and the pyruvate which has accumulated disappears, growth beginning when the pyruvate concentration in the medium has fallen back to about $10 \mu \mathrm{g} . / \mathrm{ml}$.

When the thiamine-deficient organisms are resuspended in fresh medium with nicotinic acid, they must synthesize thiamine before growth can occur. When thiamine is added, it can quickly be taken up by the thiamine-deficient organisms, and concentrations of thiamine in the organisms similar to those found in cultures growing with excess nicotinic acid are reached within a short time, perhaps seconds or at most a few minutes, even on exposure to low external concentrations of thiamine. At least some of this added thiamine is quickly converted to a functional form, as shown by the rapid decrease in pyruvate concentration in the medium. It seems that the thiamine-deficient organisms when supplied with sufficient nicotinic acid can promptly phosphorylate added thiamine.

The pyruvic oxidase of Proteus vulgaris described by Stumpf (1945) was apparently a complex consisting of a specific protein, diphosphothiamine (cocarboxylase) and a bivalent metal probably magnesium. The intact organisms contained a phosphatase which split diphosphothiamine to thiamine. Moyed $\&$ O'Kane (1956) showed that in $P$. vulgaris pyruvate was oxidized by a soluble cocarboxylase-dependent dehydrogenase which was coupled with an auto-oxidizable cytochrome system; coenzyme $\mathbf{A}$, orthophosphate, lipoic acid and flavine-adenine-dinucleotide were not involved. Neither Stumpf nor Moyed \& O'Kane found that coenzyme I was necessary for pyruvate oxidation. The strains used in the present investigation had the same cytochrome components as those described by Moyed \& O'Kane. The thiamine concentrations in the organisms which were necessary for optimal pyruvate utilization 
have been found, but the cocarboxylase concentrations in the organisms were not measured. Even with added thiamine or cocarboxylase, washed suspensions of nicotinic acid-deficient $\boldsymbol{P}$. vulgaris have a low pyruvic oxidase activity. Perhaps in the whole organisms the cocarboxylase-dependent system can link with a cozymase-dependent system. It is possible, too, that in the intact organisms there is a continuous breakdown of cocarboxylase to thiamine by the phosphatase present, and that in the absence of sufficient nicotinic acid the resynthesis of cocarboxylase is impaired. Added cocarboxylase does not, however, prevent pyruvate accumulation during the linear growth phase, and itself produces only a slight increase in rate of pyruvate oxidation by older organisms.

Thanks are due to Miss L. Hopkinson and Mr R. W. Warner for technical assistance in this work. Dr D. Herbert kindly supplied a pure sample of pyruvic 2:4-dinitrophenylhydrazone.

\section{REFERENCES}

Altmann, S. M., Crook, E. M. \& Datta, S. P. (1951). Paper chromatography of keto acids. Biochem. $J .49$, lxiii.

Barton-Wright, E. C. (1946). Practical Methods for the Microbiological Assay of the Vitamin B Complex and Essential Amino Acids. London: Ashe Laboratories Ltd.

$\mathrm{J}_{\mathrm{ACKsON}}$, F. L. (1952a). Some aspects of the growth and metabolism of Proteus vulgaris with limiting concentrations of nicotinic acid. J. gen. Microbiol. 6, vi.

JACKson. F. L. (1952b). Pyruvate accumulation and development of aneurin deficiency in Proteus vulgaris grown with limiting concentrations of nicotinic acid. IIe Congrès International de Biochimie, Paris; Resumés des Communications, p. 83.

JACKson, F. L. \& Copping, A. M. (1952). Some aspects of the growth of Proteus vulgaris with limiting concentrations of nicotinic acid. J.gen. Microbiol. 7, 107.

Lu, G. D. (1939). Studies on the metabolism of pyruvic acid in normal and vitamin $\mathbf{B}_{1}$-deficient states. I. A rapid, specific and sensitive method for the

Moyed, H. S. \& O'Kane, D. J. (1956). Enzymes and coenzymes of the pyruvate oxidase of Proteus. J. biol. Chem. 218, 831.

Stumpf, P. K. (1945). Pyruvic oxidase of Proteus vulgaris. J. biol. Chem. 159, 529.

Umbreit, W. W., Burris, R. H. \& Stauffer, J. F. (1949). Manometric Techniques and Tissue Metabolism. Minneapolis: Burgess Publishing Co.

(Received 31 December 1956) 